\title{
Desempeño económico e industrialización en Aguascalientes, 1970-1993
}

ARNOLDO ROMO VÁZQUEZ

Departamento de Economia/UAA

\section{Antecedentes históricos}

Al territorio que hoy conforma el estado de Aguascalientes formaba parte de La Gran Caxcana, ubicándose en la frontera entre las dos grandes regiones en que suele dividirse el espacio geoeconómico prehispánico: Aridoamérica y Mesoamérica. Lo que hoy es Aguascalientes pertenecía a Aridoamérica, por sus características climáticas y su aridez. ${ }^{1}$

En el primer período de conquista, Aridoamérica no fue objeto de interés ni de exploración por parte de los españoles ya que, aunado al ambiente climático relativamente inhóspito, la zona era poblada por tribus nómadas genéricamente llamadas "chichimecas" por los conquistadores, que estaban adaptadas a las posibilidades de sobrevivencia del territorio, practicando la caza y la recolección. Además, eran grupos muy belicosos, que cuando se presentó la necesidad defendieron con gran valor su territorio.

Una vez que se cubrieron las metas de conquista, sometimiento y explotación de los pueblos civilizados de Mesoamérica, así como de los recursos económicos de dicho territorio, los españoles vieron

1. Historia Mínima de Aguascalientes, INEA, México, 1991. 
la necesidad de reorientar el proceso de conquista hacia los pueblos y recursos del territorio aridoamericano.

Nuño de Guzmán, al ser destituido de la Primera Real Audiencia, fue comisionado para explorar la zona norte y occidente de lo que entonces era la Nueva España. En 1529, encargó a Pedro Almíndez Chirinos y a Cristóbal de Oñate la realización de esa tarea, estableciendo el sistema de encomienda en todo el territorio sometido. Poco tiempo después, en 1546, Juan de Tolosa descubrió en el Cerro de la Bufa las primeras minas de plata en la región, mismas que dieron origen a Zacatecas, extensa jurisdicción territorial que incluía Aguascalientes ${ }^{2}$. El paso siguiente fue la apertura de caminos entre la ciudad de Zacatecas y la capital novohispana, lo cual suponía un complicado sistema de seguridad que incluía cuarteles, fuertes y villas, cuya función principal era resguardar de todo pillaje los envíos del mineral. Fue así como nació la ruta de la plata, también llamada camino de tierra adentro.

Como parte de esta ruta se fundó la villa de Nuestra Señora de la Asunción de las Aguascalientes, el 22 de Octubre de 1575, al sur de Zacatecas, quedando bajo la jurisdicción del Reino de Nueva Galicia. Aunque la villa era un asentamiento relativamente alejado de la ruta de la plata, puesto que dicho camino pasaba por el noreste de la misma. Durante el auge minero de Zacatecas, a las haciendas situadas dentro de la jurisdicción de la villa de Aguascalientes les correspondió suministrar animales de tiro para las minas, así como granos para la población. Por otro lado, la villa progresó porque era un nudo importante en la comunicación entre la Nueva Galicia (particularmente Guadalajara) y el principal fundo minero del noroccidente novohispano, Zacatecas.

Desde la fundación de Aguascalientes, la economía regional se vinculó con actividades de comercialización de mercancías y productos provenientes del Bajío. En 1604 nació la industria artesanal

2. Ibid., p. 38.

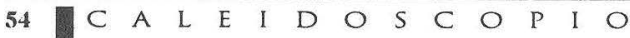


que producía artículos de lana, hierro y madera, consolidándose la actividad comercial de la villa, que era favorita de los comerciantes por ser el punto intermedio entre los principales centros mineros coloniales: Zacatecas y Guanajuato. ${ }^{3}$ Las actividades comerciales permitieron el desarrollo de la villa al conformar un nudo de comunicación entre dos caminos, la ruta de la plata y el camino entre Guadalajara y Zacatecas. La ruta de la plata o camino real de tierra adentro se abrió en 1550 y con el tiempo se transformó en el eje de comunicación entre la ciudad de México y Nuevo México, siendo una vía básica para la conquista y colonización del norte de México. El otro camino, el que comunicaba a Guadalajara con Zacatecas, pasando por Teocaltiche, fue fundamental para el establecimiento del poderío económico y político de los españoles en el norte del Bajío ${ }^{4}$.

La villa de Aguascalientes y su región también participaron del auge minero cuando, en el siglo XviII, se descubrió y explotó el distrito minero del Real de Asientos de Ibarra, cuyas posibilidades fueron encomiadas por el barón de Humboldt. La principal función de este distrito minero (que comprendía los actuales municipios de Asientos y Tepezalá) fue la de suministrar sulfato de cobre o "magistral", que era un insumo necesario para el beneficio de la plata en Zacatecas.

En términos generales, la evolución económica alcanzada por la villa y su área de influencia durante la colonia, se mantuvo igual hasta finales del siglo XIX, cuando ya Aguascalientes había dejado de pertenecer a Zacatecas y se había convertido en entidad federativa. A fines del siglo XIX suceden dos hechos de suma importancia económica. El primero es la llegada de las vías férreas, que pretendían conectar a la ciudad de México con la frontera norte del país, y la instalación de los talleres del Ferrocarril Central Mexicano en

3. Aguascalientes. Notas sobre su historia económica, COPRODEA/Gobierno del Estado de Aguascalientes, 1975. p. 3 y 4.

4. Peter J. Bakwel, Minería y Sociedad en el México Colonial: Zacatecas (15461700), Madrid, Fondo de Cultura Económica, 1976, pp. 36-40. 
la ciudad de Aguascalientes. El segundo es la apertura de la Gran Fundición Central Mexicana, propiedad de la familia Guggenheim y luego incorporada a la poderosa American Smelting and Refining Company (ASARCO).

Dada la orientación "hacia afuera" del modelo porfirista, el ferrocarril buscaba integrar al país y hacer de él un centro en el tránsito de mercancías entre Europa y Asia (pretendiendo cierto desarrollo regional). La introducción de este medio de comunicación alteró los equilibrios regionales heredados de la colonia, ya que sólo las principales ciudades del centro del país, de la frontera norte y el puerto de Veracruz, fueron las realmente beneficiadas, debido a que se acortó la distancia entre los centros de producción de excedente económico y los de distribución e intercambio. ${ }^{5}$

En este contexto se tendió la línea ferroviaria que unía a la ciudad de Aguascalientes con la de México y, a partir de 1890, con San Luis Potosí. Esto trasciende en la vida del comercio, minería e industria del estado.

La introducción del ferrocarril modificó radicalmente el sistema urbano del centro del país. Según Kemer y Royce, ${ }^{6}$ la instalación de las vías férreas benefició a las ciudades unidas a la capital del país y a los puertos de importancia, pues suscitó un cambio histórico en el patrón territorial colonial. Decayeron las ciudades ligadas al camino de tierra adentro (Tepezalá en Aguascalientes; Ojuelos en Jalisco y San Felipe, Dolores y San Miguel en Guanajuato) y surgieron 0 se fortalecieron las ciudades por donde pasaba el ferrocarril: Salamanca, Irapuato y León en Guanajuato; y las ciudades de Aguascalientes y Zacatecas. Para Aguascalientes, su inserción en la vía del ferrocarril significó un salto en su crecimiento econó-

5. Alejandra Moreno Toscano y Enrique Florescano, El sector externo y la organización espacial y regional de México, 1521-1910, Puebla, Universidad Autónoma de Puebla, 1977, p. 57.

6. R. V. Kemer y A. P. Royce, "La urbanización mexicana desde 1821", Relaciones, núm. 7, 1981, pp.6-7. 
mico, pues fortaleció las actividades históricas del comercio, la minería -en sus fases de extracción y de beneficio-, y la industria, al instalarse los talleres de reparación del Ferrocarril Central.

Una de las características de la historia industrial de la entidad en el siglo Xx es el hecho de que la evolución del sector ha estado ligada a la presencia de una empresa. Durante las primeras décadas del siglo el comportamiento del sector y de la economía en su conjunto, es explicado por la fundición de la ASARCO, la cual, al ser reubicada en San Luis Potosí en 1925, provocó una gran depresión económica. Tal depresión fue superada en los años treinta, cuando la industria alimentaria sustituyó a la minerometalúrgica como industria clave, gracias a una planta procesadora de trigo y maíz (La Perla, abierta desde principios de siglo), y al iniciarse la vitivinicultura. Durante los años cuarenta se iniciaron las primeras empresas textiles de la entidad, actividad que llegaría a verse como un signo distintivo de la industria manufacturera local a nivel nacional, hasta finales de los años setenta. Sin embargo, a partir de la década de los ochenta, el sector industrial fue sometido a una radical transformación en su estructura, dinámica y consecuencias socioeconómicas.

Otro hecho importante para la consolidación del sistema urbano y socioeconómico de la zona norte del país fue la construcción de la carretera panamericana a fines de los años cuarenta. Esta vía de comunicación complementó al sistema ferroviario y fortaleció la vocación económica relativamente diversificada de la entidad, en las actividades de comercio de abarrotes de alcance regional. Complementó también el desenvolvimiento económico estatal, junto con las actividades agropecuarias propias del semidesierto: la agricultura de temporal y la ganadería.

El desenvolvimiento económico de la entidad, a lo largo de su historia, se ha sostenido gracias a relevos intersectoriales. La economía estatal ha tenido como actividad permanente al comercio, aunque en pocas ocasiones como sector clave de la economía. 
La industria ha presentado comportamientos cíclicos de fuerte impacto para la economía y la sociedad locales. En la década de los cuarenta, la industria experimentó un auge que declinó a finales de dicha década, dando lugar a una situación deprimente que sólo se vio atenuada por el comercio y la actividad agrícola. A partir de los años cincuenta y hasta finales de los sesenta se vivió una fase prolongada de desindustrialización relativa, pues el sector secundario pasó de una participación en el PIB total cercana al 60 por ciento en 1940, al 25 por ciento en 1970 .

Por tanto, durante todo el período de vigencia y auge del modelo sustitutivo de importaciones, la economía estatal se vio alejada de la obtención de posibles beneficios, dado que la inversión industrial, que deseaba instalarse en el centro-norte del país, era siempre atraída por las aglomeraciones industriales de Jalisco y Nuevo León. Se generó así un círculo vicioso para la economía local consistente en que no era posible la industrialización en Aguascalientes porque no poseía la industria suficiente como para generar economías externas y de aglomeración buscadas por la nueva inversión. La necesaria transformación del capitalismo nacional, que implicó a su vez una distinta industrialización, favoreció a la entidad al radicarse en ella la suficiente masa de inversión nacional y extranjera como para romper el círculo vicioso de la desindustrialización.

\section{EL DESEMPEÑO ECONOMICO RELATIVO, 1970-1993}

La evolución de la economía estatal puede valorarse de modo más adecuado si observamos su desempeño en comparación con el desempeño nacional. ${ }^{8}$

7. Arnoldo Romo, Un patrón de industrialización para el estado de Aguascalientes, Tesis, ENEP-Acatlán, LNAM, 1985, p. 94 y ss.

8. En este apartado adaptamos la propuesta analítica de la OCDE que se utiliza para dimensionar y comparar el comportamiento de economías de muy diverso nivel de desarrollo. A la economía bajo estudio se le evalúa por los valores alcanzados por 
El desempeño se puede medir metodológicamente por la interacción de los contenidos en los factores de tamaño económico, el cual toma en cuenta el monto de la población total, la participación del Producto Interno Bruto (PIB) estatal en el Producto nacional y la superficie territorial de la entidad; la estructura económica, que contempla las participaciones porcentuales de la población urbana, de la población económicamente activa (PEA), de la PEA industrial y de la población subempleada en la población total; el desempeño económico, que se identifica mediante el producto per cápita, la relación entre el PIB y la PEA total, la productividad industrial y la productividad total de la economía; finalmente el resultado económico, que se mide por la relación de desigualdad en la distribución familiar del ingreso y por el monto del índice de bienestar socioeconómico de la entidad respecto al país.

\section{Tamaño económico relativo}

A pesar de que territorialmente Aguascalientes es uno de los estados más pequeños del país, desde 1970 hasta la fecha, ha aumentado su presencia y protagonismo en el contexto nacional. La entidad ocupa sólo 3 por ciento del territorio nacional y, hasta 1993, lo habitaba cerca del 1 por ciento de la población del país. Sin embargo, la densidad poblacional ha sido tradicionalmente más alta y ha crecido a mayor velocidad que la nacional, como puede verse en el cuadro número 1 .

tres factores: el tamaño relativo, la estructura económica y el desempeño. Nosotros agregamos un factor, el de resultados, que expresa los impactos positivos o negativos, en términos socioeconómicos, de los valores alcanzados por los otros factores. En seguida construimos un Índice de Desempeño Económico Relativo para el país y para Aguascalientes, compuesto por el producto no ponderado de los valores de cada uno de los indicadores estadísticos de cada factor a través del tiempo. El monto alcanzado por el país lo igualamos a la unidad y lo comparamos con el monto estatal, logrando con ello una imagen relativa de los diversos factores del desempeño económico del estado. 


\section{Cuadro 1}

Densidad de población (personas por $\mathrm{km}^{2}$ )

\begin{tabular}{|l|l|l|l|}
\hline & 1970 & 1980 & 1993 \\
\hline PAÍS & 25 & 34 & 43 \\
\hline AGS. & 60 & 93 & 134 \\
\hline
\end{tabular}

Fuente: Cálculo a partir del VI Informe de Gobierno de Carlos Salinas de Gortari (1994).

En el limitado territorio del estado, sin embargo, se ha desarrollado un esfuerzo productivo que se ha destacado por un aumento del producto económico estatal en el producto nacional. En 1970 el estado tenía un aporte del 0.5 por ciento del producto nacional, en 1980 subió al 0.6 por ciento y para 1993 la participación fue del 0.8 por ciento. Estos datos no reflejan, sin embargo, el crecimiento del tamaño económico estatal; si lo relativizamos en términos de producto por $\mathrm{km}^{2}$, observaremos la dimensión de la economía, tanto estatal como nacional.

En el cuadro 2 puede verse que, en 1970, el país produjo 263 pesos por unidad de superficie, mientras que el estado producía un poco más del doble: 510 pesos por $\mathrm{km}^{2}$. El producto estatal por unidad de superficie fue superior en todo momento al alcanzado por el país. La nación aumentó su dimensión económica en un 57 por ciento a lo largo del período 1970-1993, en tanto que Aguascalientes lo hizo en un 73 por ciento. Puede observarse también que al inicio del período, entre 1970 y 1980, el crecimiento de ambas economías fue muy semejante, en torno al 50 por ciento, mientras que, en la etapa final del período, 1980-1993, la nación crece al 11 por ciento, mientras que la entidad lo hace al 38 por ciento.

Como veremos más adelante, este comportamiento divergente obedece a la influencia de los modelos de desarrollo económico presentes a lo largo del período. Entre 1970 y 1980 se vivió la cri- 
sis del modelo sustitutivo de importaciones, que venía dinamizando a la economía nacional desde de los años cuarenta. En cambio, como lo hemos mencionado, la economía local fue una de las que quedó al margen de los beneficios de dicho modelo. Sin embargo, a partir de los años ochenta Aguascalientes logró anticipar la lógica de funcionamiento del nuevo capitalismo mexicano $y$, mediante una acelerada industrialización exportadora, mejoró su dimensión económica relativa.

\section{Cuadro 2}

PIB por $\mathrm{km}^{2}$ (miles de pesos de 1994)

\begin{tabular}{|l|l|l|l|}
\hline & 1970 & 1980 & 1993 \\
\hline PAÍS & 263.00 & 547.00 & 616.00 \\
\hline AGS. & 510.00 & $1,170.00$ & $1,899.00$ \\
\hline
\end{tabular}

Fuente: Cálculo a partir del VI Informe de Gobierno de Carlos Salinas de Gortari, 1994, p. 509.

Si conjuntamos todos los elementos que nos permitan dimensionar a las economías estatal y nacional, a través de un índice (ver cuadro número 3), podremos observar que en los años setenta el tamaño relativo de la economía estatal era 50 por ciento más pequeña que la nacional; para 1980, era sólo un 30 por ciento menor y para el fin del período, la economía estatal era ya 2.2 veces más grande que la nacional. Esto se debe a que el tamaño relativo del país vio decaer su alto ritmo de crecimiento, al pasar del 11 por ciento anual en los años setenta, a sólo 2.6 por ciento anual en la fase final del período, mientras que lo contrario sucedió con el tamaño de la economía estatal, que pasó de un crecimiento del 4 por ciento en los años setenta, a cerca del 11 por ciento en los siguientes trece años. 


\section{Cuadro 3}

Tamaño Económico Relativo

\begin{tabular}{|c|c|c|c|c|c|}
\hline \multicolumn{4}{|c|}{ Valor del Índice } & \multicolumn{2}{c|}{ TMAC\% } \\
\hline & 1970 & 1980 & 1993 & $70-80$ & $80-93$ \\
\hline PAÍS & 1.000 & 1.000 & 1.000 & 11.2 & 2.6 \\
\hline AGS. & 0.531 & 0.788 & 2.207 & 4.0 & 10.8 \\
\hline
\end{tabular}

Fuente: Cálculo a partir del VI Informe de Gobierno de Carlos Salinas de Gortari, 1994, p. 509.

\section{Estructura Económica Relativa}

El comportamiento del tamaño económico relativo se basa en los rasgos estructurales relativos que a continuación se presentan. La estructura económica relativa se refiere a la forma en que se relacionan distintos aspectos de la población, como la urbana, la económicamente activa y la ocupada en la industria, tanto en el país como en el estado.

El cuadro 4 muestra un monto mayor de población urbana en el estado que en el país. Sin embargo, el país presenta un ritmo más intenso de crecimiento en este mismo aspecto. En el estado se registra una estabilización relativa, sobre todo en la década final del período.

\section{Cuadro 4}

Participación porcentual de la población urbana

\begin{tabular}{|c|c|c|c|}
\hline & 1970 & 1980 & 1993 \\
\hline PAÍS & 58.70 & 66.30 & 71.30 \\
\hline AGS. & 63.60 & 70.40 & 76.50 \\
\hline
\end{tabular}

Fuente: Cálculo a partir del VI Informe de Gobierno de Carlos Salinas de Gortari, 1994, p. 509. 
Además, los montos de participación de la población económicamente activa (PEA) en el total, son ligeramente superiores en la nación, aunque con una tendencia descendente en la parte final del período, luego de alcanzar su máximo en 1980. En Aguascalientes se registra un crecimiento moderado (14 por ciento) entre 1970 y 1980, para después estabilizar la proporción de activos en su población.

\section{Cuadro 5}

Part. porcentual de la PEA en el total de la población

\begin{tabular}{|c|c|c|c|}
\hline & 1970 & 1980 & 1993 \\
\hline PAÍS & 26.90 & 32.00 & 28.80 \\
\hline AGS. & 25.70 & 29.90 & 29.20 \\
\hline
\end{tabular}

Fuente: Cálculo a partir del VI Informe de Gobierno de Carlos Salinas de Gortari, 1994, p. 515.

Es notorio que, en ambas economías, la participación de la población activa es relativamente baja, por lo que la tasa de dependencia aparece como un obstáculo estructural muy importante.

Otro elemento estructural de importancia es la participación de la PEA ocupada en la industria (cuadro 6). La nación presenta una tendencia relativamente estable en este punto, ya que a lo largo del período la PEA participó en promedio con un 23 por ciento, al tiempo que en Aguascalientes la población industrial vió crecer su participación de manera sostenida, ya que casi duplicó la proporción de activos en las actividades secundarias, denotando así la fuerte transformación de su estructura económica.

En cuanto a los montos de participación de la PEA con ocupación no especificada, la proporción es muy semejante en ambas economías: baja en 1970, alta en 1980 (en torno al 30 por ciento) y nuevamente mínima en 1993. 
Cuadro 6

Participación porcentual del PEA industrial

\begin{tabular}{|c|c|c|l|}
\hline & 1970 & 1980 & 1993 \\
\hline PAÍS & 24.30 & 22.90 & 28.70 \\
\hline AGS. & 23.10 & 31.40 & 56.70 \\
\hline
\end{tabular}

Fuente: Cálculo a partir del VI Informe de Gobierno de Carlos Salinas de Gortari, 1994, p. 515.

Los rasgos estructurales relativos de la economía estatal han evolucionado de acuerdo con el crecimiento del tamaño de la misma. En el cuadro 7 observamos que la estructura económica de Aguascalientes fue incrementando su composición con respecto a la del país: de ser ligeramente inferior a ella, la rebasó en 1980 (1.36 puntos) y finalmente la duplicó en 1993. Esto se debió principalmente a la gran participación de la PEA urbana e industrial en la economía local.

La estructura de la entidad presentó un fuerte dinamismo en su transformación, permitiendo un rápido proceso de industrialización. Por tanto, el crecimiento de la estructura económica relativa de Aguascalientes es superior en todo tiempo a la nacional, sobre todo a partir de la década de los ochenta, cuando la tasa media de crecimiento anual pasó del 2.4 al 1.5 por ciento, en tanto que la de Aguascalientes se mantuvo arriba del 5 por ciento.

\section{Cuadro 7}

Estructura Económica Relativa

\begin{tabular}{|l|l|l|l|l|l|}
\hline \multicolumn{3}{|c|}{ Estructura } & \multicolumn{2}{c|}{ TMAC\% } \\
\hline & 1970 & 1980 & 1993 & $70-80$ & $80-93$ \\
\hline PAÍS & 1.000 & 1.000 & 1.000 & 2.4 & 1.5 \\
\hline AGS. & 0.986 & 1.360 & 2.143 & 5.7 & 6.7 \\
\hline
\end{tabular}




\section{Desempeño Económico Relativo}

El factor de desempeño conjuga la conducta productiva de la población, en términos globales y sectoriales, a través de la revisión de su productividad.

El producto per cápita (el producto total entre la población total) registra montos superiores en la nación, aunque con tendencia descendente en la parte final del período. La entidad, por su parte, muestra un crecimiento sostenido en el esfuerzo productivo, llegando a igualar al producto per cápita nacional en 1993.

Cuadro 8

PIB per cápita (miles de pesos de 1994) PIB total/personas

\begin{tabular}{|c|r|r|l|}
\hline & 1970 & 1980 & 1993 \\
\hline PAÍS & 10.66 & 16.03 & 14.47 \\
\hline AGS. & 8.45 & 12.61 & 14.15 \\
\hline
\end{tabular}

Fuente: Cálculo a partir del VI Informe de Gobierno de Carlos Salinas de Gortari, 1994, p. 509 y 531.

En cuanto a la productividad total, considerando la relación entre el producto y la población activa de la sociedad, las tendencias de compörtamiento indican que en el país la productividad pasó de cerca de 39.6 mil pesos por persona en 1970, a un rendimiento estable de la población en tormo a los 50 mil pesos a lo largo de la siguiente década.

\section{Cuadro 9}

Productividad total (miles de pesos de 1994) (PIB total/PEA total)

\begin{tabular}{|c|c|c|c|}
\hline & 1970 & 1980 & 1993 \\
\hline PAÍS & 39.69 & 50.10 & 50.18 \\
\hline AGS. & 32.86 & 42.17 & 48.51 \\
\hline
\end{tabular}

Fuente: Cálculo a partir del VI Informe de Gobierno de Carlos Salinas de Gortari, 1994, p. 509 y 531. 
El rendimiento productivo de la población activa en la entidad fue creciente en el período de estudio, con una tendencia de acercamiento a los montos nacionales. La diferencia de productividad entre ambas economías era de seis mil pesos en 1970 y se redujo a sólo 1.6 mil pesos en 1993. Sin embargo, las diferencias en la productividad entre las dos economías permanece si las revisamos desde una perspectiva sectorial. La productividad industrial del país pasó de 41 mil pesos en 1970 a 56 y 53 mil de pesos en 1980 y 1993 respectivamente. Mientras que el sector industrial local pasó de 19 mil pesos a 29 y 38 miles de pesos en los años respectivos.

A diferencia de los factores anteriores, en los cuales la posición que guarda el estado es mejor que la nacional, el rubro del desempeño no es satisfactorio para la entidad, pues aumentó el ritmo de desempeño, en términos absolutos, de las dos economías, en el transcurso del tiempo. En términos dinámicos, el desempeño relativo de Aguascalientes presenta una tendencia de mejoría dado que su crecimiento es superior al 7 por ciento entre 1980 y 1993, en contraste con el estancamiento en el desempeño económico nacional, que es de sólo el 1 por ciento en el mismo lapso.

\section{Resultados socioeconómicos relativos}

La mejor forma de valorar el desenvolvimiento de un organismo económico -además de reconocer su dimensión, estructura y fun-

\section{Cuadro 10}

Desempeño Económico Relativo

\begin{tabular}{|l|c|c|c|c|l|}
\hline \multicolumn{3}{|c|}{ Desempeño } & \multicolumn{2}{c|}{ TMAC\% } \\
\hline & 1970 & 1980 & 1993 & $70-80$ & $80-93$ \\
\hline PAÍS & 1.000 & 1.000 & 1.000 & 11.2 & 2.6 \\
\hline AGS. & 1.369 & 0.264 & 0.616 & $-2,7$ & 7.8 \\
\hline
\end{tabular}


cionamiento- es la de reconocer el impacto positivo o negativo de todos estos elementos sobre la sociedad que participa en dicho organismo.

La manera en que valoraremos las economías bajo análisis será a través de sus resultados socioeconómicos, identificados a través del nivel de desigualdad en la distribución del ingreso familiar y a través del grado de bienestar alcanzado por ellas.

El nivel de desigualdad, obtenido por la relación entre los estratos más pobres y los más ricos, indica el número de veces en que el ingreso de los más ricos supera al de los más pobres.

Con base en el cuadro 11 puede decirse que la desigualdad es más profunda en el país ya que, aunque ha venido descendiendo a lo largo del período, aún se requiere un promedio de diez veces más, el nivel de ingreso del 20 por ciento más pobre de la población para igualar el monto de ingreso del 20 por ciento más rico. La participación porcentual de los más pobres en el ingreso no ha alcanzado el 5 por ciento del ingreso, mientras que los más ricos absorben más del 55 por ciento del mismo.

Cuadro 11

Relación de desigualdad en el ingreso familiar (Deciles IX+X/I+II)

\begin{tabular}{|l|c|c|c|r|r|r|}
\hline & \multicolumn{3}{|c|}{ México } & \multicolumn{3}{c|}{ Aguascalientes** } \\
\hline Deciles & $1968^{*}$ & $1977^{*}$ & $1992^{* *}$ & 1970 & 1980 & 1990 \\
\hline I+II & 3.40 & 3.50 & 4.30 & 4.70 & 4.90 & 5.80 \\
\hline IX+X & 58.40 & 58.40 & 53.70 & 54.26 & 49.40 & 49.10 \\
\hline Relación & 17.18 & 16.69 & 12.49 & 11.54 & 10.08 & 8.47 \\
\hline
\end{tabular}

Fuente: $\left({ }^{*}\right)$ M. Camberos, La desigualdad y el crecimiento en México, México, FCE-UNAM, 1994.

(**)INEGI, Encuesta de Ingresos y Gastos, Aguascalientes, 1992.

$(* * *)$ Y. Padilla y G. Huerta, "¿Avanzamos hacia la equidad?" en C. Vargas, Aguascalientes en los noventa, estrategias para el cambio, Aguascalientes, ICA, 1993. 
En Aguascalientes se observan niveles relativamente inferiores a los registrados en el país, con montos de participación de los pobres cercanos al 5 por ciento y de los ricos un poco inferior al 50 por ciento.

Un aspecto destacable en cuanto al desempeño de las dos economías consiste en que, si bien el país ha presentado un mejor desempeño económico, éste no se ha difundido en términos de bienestar para la población nacional, es decir, los frutos de la productividad se han concentrado en provecho de la población más rica. A nivel local, no obstante el inferior desempeño productivo de la economía, el ingreso ha podido distribuirse relativamente mejor que en el nivel nacional.

La inequitativa distribución del ingreso influye en la desigualdad de los niveles de bienestar socioeconómico de las dos economías. El cuadro 12 revela cómo el nivel de bienestar nacional, si bien es creciente, durante los años finales del período tiende a estancarse, en tanto que para Aguascalientes se registra un salto cualitativo muy importante a partir de 1980, al duplicarse el valor del índice de bienestar.

\section{Cuadro 12}

Bienestar social (valor de Índice)

\begin{tabular}{|l|c|l|l|}
\hline & Nacional (a) & Ags.(b) & Relación:(a/b) \\
\hline 1970 & 144 & 127 & 0.88 \\
\hline 1980 & 181 & 203 & 1.12 \\
\hline 1990 & 187 & 214 & 1.14 \\
\hline
\end{tabular}

Fuente: BAnamex, Examen de la situación económica de México, número 832, 1995, p. 82. 
Si comparamos los valores del índice de las dos economías tenemos que Aguascalientes supera en 1.1 veces al país durante el período 1980-1993.

La combinación de los niveles de desigualdad distributiva y los índices de bienestar permiten formar una imagen clara del comportamiento de las economías en términos de cobertura de los objetivos socioeconómicos en ambos sistemas.

Vemos en el cuadro 13 que los resultados son satisfactorios para el estado, ya que han crecido y son superiores a los alcanzados por la nación y han tendido a estabilizarse a partir de los años ochenta.

\section{Cuadro 13}

Resultado Económico Relativo

\begin{tabular}{|l|l|l|l|l|l|}
\hline \multicolumn{3}{|c|}{ Resultado } & \multicolumn{2}{c|}{ TMAC\% } \\
\hline & 1970 & 1980 & 1993 & $70-80$ & $80-93$ \\
\hline PAÍS & 1.000 & 1.000 & 1.000 & 2.0 & 2.5 \\
\hline AGS. & 1.312 & 1.856 & 1.688 & 6.2 & 1.8 \\
\hline
\end{tabular}

Por lo tanto, la economía de Aguascalientes ha sabido aprovechar su tamaño, estructura y funcionamiento económicos, traduciéndose, a lo largo del período, en un resultado positivo y superior al obtenido por el país.

$\mathrm{Al}$ conjuntar las tasas de crecimiento de cada índice particular (de los cuadros 3, 7, 10, y 13) obtenemos que, en términos globales, el país creció en su Índice Total de Desempeño Relativo un 7.6 por ciento entre 1970 y 1980 y sólo un 1.9 por ciento entre 1980 y 1993, mientras que Aguascalientes creció un 3.3 y un 6.8 por ciento en los lapsos respectivos. Con esto reconocemos que el comportamiento de la economía estatal es divergente al del país, dado que muestra un ritmo anticíclico con respecto a él. 


\section{LA INDUSTRIALIZACIÓN DE AGUASCALIENTES Y LA REESTRUC- TURACión DEL CAPITALISMO MEXICANO}

La década de los años ochenta es tan importante en el tiempo mexicano como lo fueron las décadas de los años diez y cuarenta, ya que ellas marcaron puntos de inflexión intensos en la trayectoria histórica. Podemos afirmar esto, porque la segunda década del siglo se caracterizó por la consolidación del Estado nacional que, al surgir de un movimiento armado que contenía fuerzas sociales con intereses de grupo muy particulares, otorgó al país un proyecto (de carácter nacionalista) más o menos diferenciable de los del resto de América Latina. Los años cuarenta se caracterizaron por la consolidación de la base material que el proyecto nacional requería para su viabilidad: una economía basada en la fortaleza y la autosuficiencia industrial.

Estos dos hechos confirieron a México una mediana posición en el concierto internacional, al ser poseedor de un estado nacional fuerte y de un proyecto económico bien definido, abocado al abastecimiento del mercado interno a través de la sustitución de importaciones.

Esto rindió buenos frutos a lo largo de cuarenta años, por lo que los agentes económicos y políticos no se dieron a la tarea de revisar la evolución de las tendencias de transformación de la economía mundial, ni de adecuar el proyecto y el modelo a las nuevas rutas del contexto económico internacional.

La exigencia de cambios estaba presente desde principios de los años setenta, cuando el éxito industrial-exportador de los países asiáticos y la crisis del petróleo impactaron intempestivamente la confiada conducción política y económica de varios países, tanto industrializados como subdesarrollados. ${ }^{9}$

Los países industrializados -0 , para ser precisos, las compañías transnacionales radicadas en éstos-, a mediados de la década de

9. René Villarreal, Industrialización, deuda y desequilibrio externo en México, 1929-1988, México, Fondo de Cultura Económica, 1988.

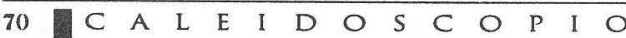


los setenta gestaron una revolución tecnológica que les permitiera lanzarse a una nueva etapa de primacía hegemónica, lo cual daría origen al fenómeno de la globalización. ${ }^{10}$

Por su parte, países como el nuestro continuaron con la inercia operativa del proyecto y modelo ya mencionados, hasta que la insuficiencia dinámica del mismo fue insoportable, traduciéndose esto en un endeudamiento implacable y en la atrofia operativa del aparato productivo.

Ante esta situación, a toda prisa y con el consiguiente costo social, los agentes económicos y políticos, nacionales y extranjeros -no todos, por cierto-, esgrimiendo discursos de modernidad y eficiencia, a mitad de los años ochenta (con una década de retraso), se dieron a la tarea de reestructurar el capitalismo mexicano.

Por ello afirmamos que los años ochenta destacan como un punto de inflexión en la trayectoria de la economía nacional, pues durante ellos se lanzó al país a un nuevo intento de inserción en la dinámica económica mundial. Esto ha implicado, hasta la fecha, un gran esfuerzo y un costo socioeconómico y político para el país.

Ahora bien, los estados nacionales son fuertemente impactados por dos tipos de fuerzas. Una externa, proveniente del influjo de la

I0. A. Dabat y M. A. Rivera proponen una definición de globalización, que nos permitimos citar por extenso: "globalización es el resultado final de un rápido proceso de internacionalización de la producción y las transacciones entre países... que conjuga: un altísimo nivel de comercio internacional en comparación de la producción mundial; un nuevo papel de las empresas transnacionales en comercio y producción; el surgimiento de una nueva división internacional del trabajo; integración de los mercados financieros internacionales y nacionales en un espacio operativo unificado, gracias a la revolución de las telecomunicaciones; elevada migración internacional de población (calificada que uniformiza relativamente) los mercados laborales nacionales; y la coordinación de políticas macroeconómicas de las grandes potencias (...). El resultado de esto, para nuestros países, es que se debilita la centralidad de los mercados nacionales como núcleo central del intercambio comercial; las empresas transnacionales se ven forzadas al establecimiento de alianzas estratégicas y los estados nacionales a conformar bloques comerciales regionales a fin de ampliar su espacio vital y competir eficazmente". Ver "La transformación de la economía Mundial”, en Investigación Económica, no. 206, 1993, pp. 137-138. 
economía mundial globalizada, que exige a los países la preeminencia de la competitividad y la mutación en la concepción de la soberanía, para adecuarlos a esta nueva fuerza mundial y para facilitar la conformación de bloques comerciales regionales. La otra fuerza está constituida por la coincidencia de los procesos de privatización de la economía, desregulación de los mercados, desburocratización de los aparatos administrativos del gobierno y refuncionalización del poder político, creándose con todo esto una nueva relación entre el estado y el capital y entre aquél y la sociedad.

En el caso muy concreto de la economía mexicana en general y del sector industrial en particular, desde los años setenta se hizo necesario exponer a ambas instancias económicas a la competencia y concurrencia de la economía mundial, pues ya las ramas industriales habían superado la fase "infantil", en la que la monopolización de la producción era la forma más común de operar en las ramas manufactureras. Sin embargo, al prolongar la etapa proteccionista se impidió alcanzar una etapa más avanzada de industrialización. Se desalentó la inducción del progreso técnico y por lo tanto la obtención de sus frutos: el avance de las fuerzas productivas y la competitividad de las manufacturas nacionales.

La fuerza de la globalización ha exigido que la economía mexicana asuma un proceso de reestructuración en su funcionamiento, tendiente a que pueda relacionarse adecuadamente con la economía mundial. Esta adecuación persigue tres líneas modernizadoras: el restablecimiento de la rentabilidad del capital, por medio de la recuperación de la eficiencia productiva; la eliminación de la distorsión en la asignación de los recursos productivos creados por la sobre expansión y el agotamiento parcial del potencial dinámico del Estado interventor; y la integración a la economía mundial, abriendo la economía nacional para adecuar costos, precios y ganancias a las imperantes a nivel internacional. ${ }^{11}$

11. Miguel Rivera, El nuevo capitalismo mexicano, México, Era, 1992, p. 98.

72 C 
Los cambios del capitalismo mexicano se inscriben en el complejo proceso de reestructuración del capitalismo mundial. En dicho proceso se superponen factores de naturaleza económica, tecnológica, política y cultural como parte de una respuesta ofensiva y defensiva del capital mundial ante la tendencia descendente.de sus niveles de rentabilidad buscando, vía la globalización (que contiene a la reestructuración), la apertura de un nuevo ciclo de expansión, semejante al del período posterior a la Segunda Guerra Mundial.

Esta transformación implicó para México adentrarse en un nuevo estadio de industrialización, abandonando el ya no rentable esquema industrial de orientación endógena. Sin embargo, para que se materializara esto, se necesitó de un drástico cambio en el sistema productivo a través de tres vertientes: por el lado de los mercados, facilitando la competencia externa; por el lado de los procesos productivos, mediante la irrupción de nuevas formas de producción a raíz de la presencia de la tercera revolución industrial; por el lado de la organización y la gestión de la mano de obra, sustituyendo las formas verticales tayloristas-fordistas por las formas horizontales del trabajo en equipo, el sistema "justo a tiempo" y la movilidad interna. $^{12}$

Se reconoce que lo que caracteriza a la sociedad mexicana es la apertura y exposición a las nuevas fuerzas económicas (el libre cambio), culturales (nuevas redes de información), políticas (neoliberalismo) y sociales (nuevas formas de organización grupal) mundiales. En lo económico, la libre concurrencia es el catalizador de la transformación del capitalismo nacional. Se afirma que el restablecimiento de las condiciones de concurrencia (tanto de origen interno como externo) con objeto de dinamizar la economía, junto con

12. Enrique de la Garza, "Reestructuración espacial y reconversión industrial", en Mario Bassols (coordinador), Campo y ciudad en una era de transición, México, LAM Iztapalapa, 1994. 
la racionalización del intervencionismo estatal, era imperioso, por consideraciones endógenas.

La creciente internacionalización de la vida económica reforzaba esta tendencia al intensificar la lucha por el mercado mundial. Por eso, al inicio de la década de los ochenta fue necesario iniciar, en medio de una profunda crisis social, los procesos de reestructuración orientados a satisfacer exigencias que habían sido postergadas por décadas.

Por tanto, puede decirse que los rasgos más relevantes del nuevo estilo de desarrollo mexicano son: a) la conformación de un modelo manufacturero exportador; b) la posición clave de la inversión extranjera directa; c) los cambios en el comportamiento y estructura del empleo, el gasto y el ingreso público; y, d) lo importante para nosotros, una nueva localización territorial de la industria.

La reestructuración productiva implica una reestructuración espacial de la industria entendiendo esto no como la simple (re)distribución geográfica entre la industria moderna y la atrasada, sino como el surgimiento de zonas con núcleos modernizados o en reestructuración que contienden con su archipiélago atrasado. ${ }^{13}$

Lo que podemos derivar de lo anterior es que existe una espacialidad en el proceso de reestructuración. Por espacialidad entendemos la forma en que se implantan, a escala territorial, los cambios sucedidos en el modelo de acumulación nacional. ${ }^{14}$

La espacialidad implica no sólo considerar los cambios en la ubicación de la industria por regiones, sino los encadenamientos en mercados de trabajo, los factores productivos, políticos, sociales y culturales relativos a la localización y funcionamiento dinámico de la industria. Al coexistir estos factores, el espacio puede ser reestructurado.

13. Ibid., pp. 11-12.

14. Blanca Ramírez, "Modernización y reestructuración territorial", en Ciudades, número 13, 1992 y Miguel Vite, "Algunas visiones sobre la espacialidad", en Papeles de Población, números 5 y 6, 1996. 
La espacialidad es un producto social y es parte integrante de la constitución material y de la estructuración de la vida social. No puede ser entendida si no se incluye a la sociedad y a las relaciones sociales. La espacialidad significa la representación del proceso de reproducción del sistema capitalista en su dimensión más material.

Otro aspecto que debe tenerse en cuenta es el hecho de que la espacialidad de la reestructuración productiva no es uniforme en todo el territorio nacional, porque los grupos de poder son actores fundamentales del cambio, pero no todos los actores ni todas las regiones responden de igual manera a una misma política modernizadora, sino según su capacidad de respuesta y adaptación al modelo que se intenta implantar.

La desigual capacidad de respuesta obedece a que no todas las partes del espacio nacional tienen la misma capacidad de generar el excedente social que el nuevo estilo de capitalismo exige. El resultado, citando extensamente a Blanca Ramírez, es que

se originan procesos que algunos autores intentan diferenciar como antagónicos y excluyentes, pero que en realidad se conjugan diferencialmente en el territorio. Desindustrialización de algunas zonas, es decir, la exportación de actividades económicas y funciones de un consorcio a otras regiones... proceso priorizado ya no por la cercanía a los mercados, bajos costos de transporte o por ventajas naturales de localización de insumos. Ahora se buscan mejores condiciones en relación a la abundancia, menor costo, precisión o a la menor capacidad de lucha de los trabajadores. Paralelamente se da la re/neo industrialización o implantación de sectores punta, especializadas en ramas más productivas de otros países. Con ello cambian los criterios de ubicación ya que de ser geográfico-locacionales, se priorizan los económico-políticos..$^{15}$

Esta autora opina que se polarizan territorialmente cuatro regiones con procesos que las particularizan, en las cuales la reconversión

15. Véase el artículo citado de Blanca Ramírez, p. 4. 
técnica y económica permite diferenciar las tendencias actuales de producción sobre la base de las condiciones de relaciones de trabajo que se implantan en ellas:

1. Nueva industrialización en la zona norte del país (frontera norte y Aguascalientes como excepción), región que se constituye como el eje dinamizador del proceso manufacturero, ya que acepta parte de los procesos que descentralizan los países desarrollados.

2. Zona metropolitana de la Ciudad de México, con una aparente tendencia a la desindustrialización, dado el estancamiento que presenta la dinámica para la nueva localización industrial. Sin embargo realiza adecuaciones a las formas internacionalizantes, aunque de manera heterogénea y con esquemas un tanto diferentes a los de la zona norte del país.

3. Regiones petroleras del sudeste, su especialización productiva se desarrolla dinámicamente a pesar de haber dejado de ser el eje de la economía del país.

4. Región sur, con especialización en el turismo internacional, integrada a formas de producción agrícola tradicional.

La perspectiva inmediata de la distribución geográfica de los cambios en el capitalismo mexicano es aquélla en la cual las entidades federativas que puedan insertarse en el nuevo esquema de desarrollo podrán jugar al máximo sus ventajas comparativas. En contraste, toda entidad que no logre desenvolver adecuadamente esas ventajas, tenderá a hacerse más obsoleta y más pobre.

\section{UN CASO DE ESPACIALIDAD DE REESTRUCTURACIÓN CAPITALISTA}

La industrialización de Aguascalientes, junto con la de los estados que hacen frontera con los Estados Unidos, son ejemplos claros de la espacialidad de la reestructuración del capitalismo mexicano, pues sus economías industriales asumen un alto protagonismo justamente cuando el modelo industrial nacional se encuentra en una crisis profunda de inviabilidad como medio para el desarrollo económico. 
El estado se vio relativamente marginado de los beneficios de la industrialización sustitutiva de importaciones, al encontrarse exactamente en el centro de las tres zonas industriales más fuertes de este modelo: Jalisco, Nuevo León y la Zona Metropolitana de la Ciudad de México (ZMCM).

Esto significó que cualquier decisión de inversión tendiente a desconcentrar la ZMCM, y que deseara ubicarse en el interior del país, sería fuertemente atraída por las otras dos zonas dado que ahí se encontraban fuertes economías externas y de localización, condenando así las entidades cercanas a esta zona a la sub-industrialización, como fue el caso de Michoacán, Aguascalientes, Zacatecas, Durango y Nayarit, con respecto a Jalisco.

En estas entidades se desarrolló un círculo perverso de desindustrialización, ya que su sector secundario no avanzaba porque no crecía su inversión industrial y ésta no fluía hacia ellas porque no tenían una base industrial sólida.

Para el caso de Aguascalientes, la relación circular se rompió cuando, a principios de la década de los años ochenta, comenzó a haber flujos de inversión en giros distintos a los tradicionales (textiles, confección, alimentos y metalmecánica), con orígenes no nacionales y con el propósito básico de desarrollar oferta exportable hacia el mercado mundial.

Fluyó inversión hacia la industria de autopartes (Fomasa, 1980; Moto Diesel, 1981 y Nissan, 1982) y electrónica (Xerox, 1983 y Texas Instruments, 1984).

Los montos de inversión relativamente modestos a nivel nacional (ver cuadro 14), tuvieron un efecto multiplicador inusitado en Aguascalientes, ya que lograron atraer nuevos recursos y giros industriales hacia el estado, así como actividades terciarias muy diversas, en momentos en que el país se debatía en el proceso de reestructuración de su sistema industrial, tratando de orientarlo a la atención del mercado externo. 
Cuadro 14

Inversión extranjera en Aguascalientes

\begin{tabular}{|l|c|l|}
\hline Período & Millones de dólares & Porcentaje \\
\hline $1980-1985$ & $2,259.7$ & 86.3 \\
\hline $1986-1990$ & 106.7 & 4.1 \\
\hline $1990-1993$ & 64.6 & 2.4 \\
\hline $1994-1997$ & 187.7 & 7.2 \\
\hline Total & $2,618.7$ & 100.00 \\
\hline
\end{tabular}

Fuente: CEDECE, La inversión extranjera en Aguascalientes, Gobierno del Estado, 1997.

Los flujos de inversión se concentraron en el período de mayor aceleración de la industrialización local, 1980-1985, sostenida principalmente por las cinco empresas mencionadas anteriormente. ${ }^{16}$ Para los años siguientes, el ritmo de recursos disminuyó sensiblemente, a tal punto que, entre 1985 y 1993, el monto de inversión fue únicamente de 171 millones de dólares, cantidad que se logró en los últimos tres años.

El ritmo de los flujos de inversión ha estado fuertemente influido por los ritmos sexenales del poder ejecutivo estatal. El primer período del cuadro 14 corresponde al gobierno de Rodolfo Landeros Gallegos; el segundo, 1986-1992 al de Miguel Angel Barberena Vega; y el que comienza en 1993, al de Otto Granados Roldán.

El comportamiento pendular de la inversión extranjera en el estado se explica por las relaciones de cercanía o distanciamiento político del gobernador en turno con el liderazgo presidencial, esto se pudo observar cuando el gobernador Landeros, perteneciente al

16. CEDECE, El sector exportador de Aguascalientes, Gobierno del Estado de Aguascalientes, 1996. 
grupo político del presidente López Portillo, al iniciar su mandato, contaba ya con el compromiso firme de inversión en la entidad de parte de las compañías Nissan y Texas Instruments, siempre y cuando se construyera en el estado un aeropuerto con un nivel de operación, al menos, nacional. ${ }^{17}$ Gracias a esta circunstancia política se pudo romper, como ya hemos dicho, la relación circular de la desindustrialización local. En el período gubernamental de Landeros se estableció en la entidad el 14 por ciento de las empresas exportadoras, entre las cuales se encontraban las de más alto poder exportador hasta el momento actual.

Por su parte, el gobernador Barberena, a pesar de que tuvo diferencias con el presidente Salinas de Gortari, logró conservar la misma dinámica de atracción de la inversión del período anterior, ya que en ese lapso se instaló el mayor número de empresas exportadoras en el estado (ver cuadro 15). De las 122 empresas que existen en la actualidad, 54 iniciaron operaciones entre 1986 y 1993, es decir, el 44 por ciento de la base exportadora se formó en ese período.

La situación durante la gubernatura de Granados Roldán fue muy semejante a la que prevaleció durante la gestión de Landeros, dada su pertenencia al grupo político de Salinas de Gortari. Esto reforzó el ambiente socioeconómico y político favorable a los negocios en la entidad. En un lapso de tres años se crearon 14 de las empresas exportadoras (el 11 por ciento de ellas), básicamente en giros tradicionales del área textil, confección y otros.

La industrialización de la entidad ha sido un resultado temprano del nuevo modelo industrial nacional, ya que su base industrial exportadora se creó entre 1980 y 1985, momento en que el país

17. Una vez que Landeros tomó la gubernatura, se iniciaron los trabajos de construcción del aeropuerto, aunque la petición del aeropuerto ya se había hecho años antes, habiendo sido contemplada en el Plan Nacional de Desarrollo Urbano (1978) y en el Plan Nacional de Desarrollo Industrial (1979).

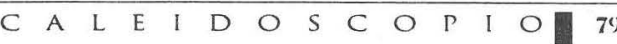


Número de empresas Exportadoras

Giros de Actividad/Año de inicio de operaciones

\begin{tabular}{|l|l|l|l|l|l|r|}
\hline Giro/Período & 1980 & $81-85$ & $86-93$ & $93-97$ & total & $\%$ \\
\hline Alimentos & 8 & 1 & 8 & 2 & 19 & 15.57 \\
\hline Textil & 6 & 0 & 2 & 1 & 9 & 7.38 \\
\hline Confección & 14 & 7 & 19 & 4 & 44 & 36.07 \\
\hline Metal Mecánica & 4 & 2 & 6 & 0 & 12 & 9.84 \\
\hline Automotriz & 1 & 3 & 3 & 1 & 8 & 6.56 \\
\hline Electrónica & 0 & 2 & 3 & 0 & 5 & 4.10 \\
\hline Otras & 3 & 3 & 13 & 6 & 25 & 20.49 \\
\hline Total & 36 & 18 & 54 & 14 & 122 & 100.00 \\
\hline Part.\% & 29.5 & 14.8 & 44.3 & 11.5 & 100 & \\
\hline
\end{tabular}

Fuente: Cálculo propio a partir de CEDECE, op. cit, 1996.

discutía la conveniencia de iniciar la reestructuración de su economía mediante una industrialización orientada al exterior.

La orientación exportadora de la economía estatal

La reestructuración del capitalismo mexicano tuvo como propósito hacer más rentable el capital asentado en su territorio 0 , en la terminología del neoliberalismo, hacerlo más competitivo. Esto significa que una economía es más competitiva si atrae capital extranjero, si es más dinámica su industria y si posee un mejor desempeño en los mercados mundiales. ${ }^{18}$

18. Juan Moreno, "La competitividad de la industria mexicana", en Fernando Clavijo y J. Casar (compiladores), La industria mexicana en el mercado mundial, Lecturas del Trimestre Económico, número 80, 1997. 
A partir de los primeros años de la década de los ochenta, Aguascalientes manifestó una aceptable capacidad de atracción de capital, tanto nacional como internacional, pero sobre todo un buen desempeño en su comercio exterior, como se tendrá la oportunidad de ver a continuación, así como un reconocido dinamismo industrial. Es decir, la industrialización de la entidad fue marcada por un alto grado de competitividad, al satisfacer estos rasgos de comportamiento.

El cuadro 16 muestra el comportamiento relativo del volumen del sector externo de la economía nacional y la de Aguascalientes. El volumen de comercio exterior [la suma de las importaciones más exportaciones $(\mathrm{M}+\mathrm{X})$ dividido entre el producto interno bruto (PIB)] representa a las unidades monetarias generadas en el comercio exterior por unidad de producto).

El cuadro describe cómo el comportamiento del sector, a nivel del país, tiende a permanecer constante en tanto que, a nivel de la entidad, se ve claramente el impacto del sector externo sobre la economía en su conjunto.

Para Aguascalientes, de una ausencia total de actividad exportadora en 1980, se obtienen valores muy importantes en 1985, que

\section{Cuadro 16}

Volumen de Comercio (M+X/P) (Millones de pesos de 1994)

\begin{tabular}{|c|c|c|}
\hline Año & Nacional & Aguascalientes \\
\hline 1980 & 0.03 & n.d. \\
\hline 1985 & 0.03 & 0.32 \\
\hline 1988 & 0.03 & 0.08 \\
\hline 1993 & 0.03 & 0.12 \\
\hline
\end{tabular}

Fuente: Cálculo propio a partir de datos de INEGI G. González Vela, Desarrollo Económico y Social de Aguascalientes, 1986-1992, ICAGobierno del Estado- Instituto Lucas Alamán, Ags., 1992. 
disminuyen en 1988 y se recuperan levemente en 1993. La disminución del volumen en 1988 se explica por el hecho de que, gracias al impacto del sector externo en el período 1980-1985, la economía en su conjunto se dinamizó de manera más que proporcional al incremento del sector externo. Por eso el PIB total creció más que dicho sector. En ese momento, a mediados de la década de los años ochenta, fue cuando se consolidaron los beneficios de la actividad exportadora de la industria, ya que ella participó con más del 90 por ciento del comercio exterior del total de la economía de Aguascalientes.

Para visualizar de manera más clara la importancia del sector externo sobre el comportamiento de ambas economías, tomemos la información de la tasa de apertura $[\mathrm{X} / \mathrm{P}]$, conformada por la relación entre volumen de exportaciones y producto.

El cuadro17 revela que la apertura es más amplia en el estado que en el país y que, además, ésta asume una tendencia creciente. Se tiene por tanto que, no obstante que en ambas economías se registra un crecimiento del producto, las exportaciones crecen de manera relativa con mayor velocidad y estabilidad en la entidad.

\section{Cuadro 17}

Tasa de Apertura y Tasa de Penetración (millones de pesos e 1994)

\begin{tabular}{|l|c|c|c|c|}
\hline & \multicolumn{2}{|c|}{ Tasa de Apertura } & \multicolumn{2}{c|}{ Tasa de Penetración } \\
\hline Año & Nacional & Ags. & Nacional & Ags. \\
\hline 1980 & 0.01 & n.d. & 0.02 & n-d \\
\hline 1985 & 0.02 & 0.07 & 0.01 & 0.25 \\
\hline 1988 & 0.02 & 0.05 & 0.02 & 0.03 \\
\hline 1993 & 0.01 & 0.06 & 0.02 & 0.06 \\
\hline
\end{tabular}

Fuente: Cálculo propio a partir del PIB por entidad federativa

82.0 C A 
$\mathrm{Al}$ analizar el elemento complementario de la tasa de apertura, la tasa de penetración de las importaciones [M/P], se observa que la economía local está más expuesta a la dependencia de las compras externas para su desempeño. Los volúmenes del indicador son superiores en Aguascalientes en todo momento, pero sobre todo cuando el volumen de comercio es más intenso (ver cuadro 17).

Los elementos señalados arriba nos muestran que la entidad desarrolló una dinámica congruente con la intención del nuevo modelo de desarrollo económico nacional: una industrialización basada en las exportaciones.

Una muestra firme de esto la podemos ver a través de la evolución del grado de industrialización ${ }^{19}$ de ambas economías.

\section{Cuadro 18}

Grado de Industrialización Nacional y de Aguascalientes, 1970-1993 (pib manufacturero/pib total)

\begin{tabular}{|c|c|c|c|c|c|c|}
\hline & 1970 & 1975 & 1980 & 1985 & 1988 & 1993 \\
\hline PAÍS & 0.24 & 0.23 & 0.23 & 0.23 & 0.34 & 0.20 \\
\hline AGS. & 0.12 & 0.14 & 0.15 & 0.28 & 0.29 & 0.20 \\
\hline
\end{tabular}

Fuente: Datos de INEGI.

El cuadro 17 muestra, paso a paso, el ritmo de participación del sector secundario en el PIB total, es decir, el grado de industrialización. Si dividimos el cuadro en los períodos 1970-1980, correspondiente al tiempo de vigencia del modelo industrializador sustitutivo, reconocemos que el grado de industrialización de la economía nacional fue muy superior al que registró el estado; y cómo, a partir de 1980-1985, fase crítica de aquella economía, Aguascalientes desarrolló una elevación súbita en su industrialización, casi dupli-

19. Héctor Soza, Planificación del Desarrollo Industrial, México, Siglo XXI, 1978, p. 11. 
cando el volumen del sector secundario, al pasar de un grado de 0.15 en 1980 a 0.28 en 1985 logrando, a partir de ese momento, sostener el ritmo industrial, ahora sí muy semejante al que registraba la nación, en torno a los 0.20 puntos en el grado de industrialización. Es decir, cuando la apertura económica de la industria se consolidó en el país, el ritmo de comportamiento y participación de la industria local asumió el mismo ritmo de aquél. Lo anterior confirma a Aguascalientes como un ejemplo temprano de la reestructuración del capitalismo mexicano.

\section{EL COMPORTAMIENTO DIVERGENTE DE LA INDUSTRIALIZACIÓN LOCAL}

Hemos afirmado que la conducta económica de Aguascalientes ha modificado su tendencia a partir de la década de los años ochenta, tanto porque ha mejorado su desempeño relativo como porque ha logrado aprovechar la amplitud de su sector externo y la presencia del capital transnacional, desarrollando un intenso proceso de industrialización. Por eso reconocemos, por un lado, que Aguascalientes es un caso de espacialidad de la reestructuración capitalista mexicana y, por otro, que observa la existencia de una trayectoria divergente en la evolución de la industria local frente a la nacional.

Una forma de cuantificar esta divergencia es a través del índice de componentes del cambio neto total $[\mathrm{CNT}] \cdot{ }^{20}$ El índice total con-

20. Una entidad federativa puede crecer bien porque tiene un sector industrial que crece más rápido que a nivel nacional o porque ella está ganando un aumento en la proporción de una o unas industrias, sin tomar en cuenta el crecimiento de las mismas. El CNT se refiere a los cambios en los indicadores estatales en dos puntos de tiempo y se concentra en saber si el cambio local es mayor o menor al nacional. El CNT mide la diferencia entre el cambios real de la entidad federativa y el cambio que se hubiera presentado si ella hubiera crecido a la misma tasa nacional.

$$
\mathrm{CNT}=\mathrm{CNP}+\mathrm{CND} \text {. }
$$

I] El Cambio Neto Proporcional. CNP $=[($ Nil/NiO) $-(\mathrm{Nl} / \mathrm{NO})] *$ EijO .

Mide el efecto de la composición industrial que proviene del hecho de que nacionalmente algunos sectores industriales crecen con mayor rapidez que otros. Así una en- 
juga, a través del cambio neto proporcional [CNP], el monto sectorial de industrias dinámicas o estancadas. Si el valor del componente es positivo la entidad posee industrias de rápido crecimiento. El factor del cambio neto diferencial [CND] muestra a través de su signo positivo si la industria de la entidad encontró ventajas locacionales superiores a las observadas en otros estados. La suma de estos componentes resumen su acción sobre la conducta del sector industrial local. ${ }^{21}$

\section{Cuadro 19}

Aguascalientes: cambio neto total

\begin{tabular}{|l|c|c|c|}
\hline Período & CNP & CND & CNT=CNP+CND \\
\hline $1970-1975$ & -70 & 1,465 & 1,395 \\
\hline $1975-1980$ & -120 & 841 & 721 \\
\hline $1980-1985$ & 144 & 10,447 & 10,591 \\
\hline $1985-1988$ & 2,900 & -299 & 2,601 \\
\hline $1988-1993$ & 1,606 & 188 & 1,794 \\
\hline
\end{tabular}

Fuente: Cálculo propio a partir de datos del INEGI.

tidad federativa que se especializa en industrias de rápido crecimiento mostrará un CNP $>0$.

II] El Cambio Neto Diferencia $C N D=[E i j-(N i l / N i O)] * E i j O$.

Aquí algunas industrias crecen en algunas regiones más rápido que en otras. Si en la entidad federativa el CND $>0$, posee ventajas locacionales mayores que relativamente a otras regiones.

III] El Cambio Neto Total $C N T=[E j-(N 1 / N O)] * E j 0$. De igual manera, puede obtenerse CNP $=$ CNT-CND $=[(\mathrm{Nil} / \mathrm{NiO})-(\mathrm{N} 1 / \mathrm{NO})]^{*} \mathrm{Eij} 0$

Nomenclatura:

Año inicial 0 , año final 1.

Eij = Tasa de crecimiento del PIB industrial (i) en la entidad federativa (j).

$\mathrm{Ej}=$ Tasa de crecimiento del PIB total en la entidad federativa (j).

$\mathrm{Ni}=$ Tasa de crecimiento del PIB industrial (i) en la nación.

$\mathrm{N}=$ Tasa de crecimiento del PIB total nacional.

21. Luis Unikel y otros, El desarrollo urbano en México, México, El Colegio de México, 1978, p. 355-356. 


\section{El cambio neto proporcional}

El cuadro 17 presenta la evolución del componente a partir de 1970 y hasta 1993. Se tienen valores negativos en la década de los setenta debido a que la industria nacional registró ritmos de crecimiento más altos que la entidad, agudizándose esto para la segunda mitad del tiempo señalado, esto como producto del círculo vicioso de la subindustrialización que ya hemos comentado.

A partir de 1980 los valores cambian de signo, reconociéndose por tanto que el ritmo sectorial local mejora respecto al nacional. En el período 1985-1988 el valor del componente es de un monto considerable a causa de que el ritmo industrial nacional presentó una de sus crisis más profundas, contrariamente a lo acontecido en el nivel local. En estos dos períodos (1980-1988), el PIB industrial nacional permaneció constante en su crecimiento, promediando el 1 por ciento, en tanto que Aguascalientes, entre 1980 y1985, registró un crecimiento industrial cercano al 16 por ciento, lo que le llevó a un cambio proporcional de -120 puntos entre 1975 y 1980; a +144 puntos entre 1980 yl 1985 y luego a 2,900 puntos en el período 1985-1988. Como vemos, a lo largo de la década de los ochenta el ritmo del cambio neto proporcional es muy intenso, mostrando que la industrialización de la entidad es distinta, en rumbo e intensidad a la nacional. Para el lapso final del período, el monto del cambio de este factor aminora relativamente su ritmo, pasando a los 1,606 puntos en el año 1993.

\section{El cambio neto diferencial}

Este componente mide la existencia y aprovechamiento de las ventajas locacionales de la entidad ligadas a la dinámica de las industrias. La información del cuadro 17 muestra que, en los años setenta, se tenían valores suficientemente atractivos para la industria pero, según el análisis anterior, dicha actividad no creció al ritmo 
que lo podía haber hecho, a causa del vacío relativo de los flujos de inversión hacia la entidad. Esto cambió dramáticamente en los años ochenta cuando, en el período 1980-1985, las circunstancias del sector se modificaron, acelerando la disponibilidad de ventajas locacionales, como lo refleja el cambio diferencial, en un monto superior a los 10,000 puntos. En los períodos siguientes, el cambio diferencial de la entidad aminora sensiblemente su ritmo, hasta hacerse negativo en el lapso 1985-1988 (-299 en relación con el período anterior); en el siguiente y último período se recupera el monto del cambio diferencial con 188 puntos. El comportamiento tendencial del cambio diferencial tiene su máximo valor en la primera mitad de los años ochenta, reflejando con ello el acelerado crecimiento en el grado de industrialización de la economía estatal.

\section{El cambio neto total}

El agregado de los dos componentes anteriores se resume en el cambio neto total (CNT). En él reconocemos que en todo momento se obtienen valores positivos, siendo más importantes en monto los registrados a partir de los años ochenta. Se observa que, en la década de los setenta, los factores locacionales (cambio neto diferencial, CND) con que cuenta la entidad se ven contrarrestados por el contenido de industrias de bajo ritmo de crecimiento (cambio neto proporcional, CNP); y cómo, a partir de los años ochenta, la dinámica industrial se incrementa fuertemente, disminuyendo los atributos locacionales como fuerza explicativa del cambio neto total. Así pues, los valores siempre positivos alcanzados por el CNT han sido soportados por la acción complementaria y alternada de los componentes.

De este modo, el salto hacia la industrialización de la economía estatal se explica por el ofrecimiento de ventajas locacionales (alto CND) más que por la posesión de industrias dinámicas; pero una vez consolidado el cambio económico de la industrialización acelera- 
da, lo que le da consistencia al CNT es el hecho de contar con industrias de alto dinamismo e impacto a nivel nacional (altos valores del CNP), cosa que sucede entre 1988 y 1993.

Finalmente, una afirmación concluyente del análisis precedente es que, en el momento en que se registran valores positivos en el CNT, a partir del instrumento metodológico utilizado, reconocemos que Aguascalientes sigue un camino relativamente distinto al nacional, por lo que sus conductas económicas son divergentes, pues el país presenta problemas críticos en su dinámica industrial, en tanto que la entidad evoluciona con buen desempeño en dicho sector.

\section{COMENTARIO FINAL}

A manera de síntesis de este artículo podemos subrayar dos hechos fundamentales para la economía estatal. Uno de ellos consiste en que el desarrollo industrial del estado, a partir de los años ochenta, presentó un funcionamiento relativamente destacado comparado con el nacional, pudiendo ser catalogado como un buen representante de la espacialidad de la reestructuración del capitalismo mexicano. El otra hecho confirma una de las tendencias de la economía local, la de asumir un comportamiento divergente, aunque cada día más tenue, respecto al ritmo de crecimiento de la economía del país. : 